\section{Rituximab plus bendamustine as front-line treatment in frail elderly ( $>70$ years) patients with diffuse large B-cell non-Hodgkin lymphoma: a phase II multicenter study of the Fondazione Italiana Linfomi}

\author{
Sergio Storti, ${ }^{1}$ Michele Spina, ${ }^{2}$ Emanuela Anna Pesce, ${ }^{3}$ Flavia Salvi,${ }^{4}$ \\ Michele Merli, ${ }^{5}$ Alessia Ruffini, ${ }^{6}$ Giuseppina Cabras, ${ }^{7}$ Annalisa Chiappella, ${ }^{8}$ \\ Emanuele Angelucci, ${ }^{9}$ Alberto Fabbri, ${ }^{10}$ Anna Marina Liberati, ${ }^{11}$ Monica Tani, ${ }^{12}$ \\ Gerardo Musuraca, ${ }^{13}$ Annalia Molinari, ${ }^{14}$ Maria Pia Petrilli, ${ }^{1}$ Carmela \\ Palladino, ${ }^{4}$ Rosanna Ciancia, ${ }^{2}$ Andrea Ferrario, ${ }^{5}$ Cristiana Gasbarrino, ${ }^{1}$ \\ Federico Monaco, ${ }^{4}$ Vincenzo Fraticelli, ${ }^{1}$ Annalisa De Vellis, ${ }^{1}$ Francesco Merli ${ }^{15}$ \\ and Stefano Luminari ${ }^{15,16}$
}

\begin{abstract}
${ }^{1}$ Department of Hematology, Universita' Cattolica Sacro Cuore Campobasso; ${ }^{2}$ Division of Oncology A, National Cancer Institute Aviano; ${ }^{3}$ Fondazione Italiana Linfomi Onlus; ${ }^{4}$ Hematology Unit, Antonio e Biagio e Cesare Arrigo Hospital, Alessandria; ${ }^{5}$ Department of Hematology, Ospedale di Circolo e Fondazione Macchi - ASST Sette Laghi, Varese; ${ }^{6}$ GRADE Gruppo Amici Dell'Ematologia; ' Unit of Hematology Ospedale Businco, Cagliari;

${ }^{8}$ Department of Hematology, Città della Salute Hospital and University, Torino; ${ }^{\circ}$ Ospedale Policlinico San Martino, Genova; ${ }^{10}$ Azienda Ospedaliera Universitaria Senese, U.O.C. Ematologia, Siena; ${ }^{11}$ Università degli Studi di Perugia, A.O.S. Maria, Terni; ${ }^{12}$ Department of Hematology, S. Maria delle Croci Hospital, Ravenna; ${ }^{13}$ Department of Hematology, IRCCS Istituto Scientifico Romagnolo per lo Studio e la Cura dei Tumori (I.R.S.T.), Meldola; ${ }^{14}$ Department of Hematology, Infermi Hospital, Rimini; ${ }^{15}$ Department of Hematology, Azienda Unità Sanitaria Locale IRCCS di Reggio Emilia and ${ }^{16}$ Department of Clinical Diagnostic and Public Health Medicine, Università degli Studi di Modena e Reggio Emilia, Modena, Italy
\end{abstract}

\section{ABSTRACT}

W e conducted a phase II study to assess activity and safety profile of bendamustine and rituximab in elderly patients with untreated diffuse large B-cell lymphoma (DLBCL) who were prospectively defined as frail using a simplified version of the Comprehensive Geriatric Assessment (CGA). Patients had to be over 70 years of age, with histologically confirmed DLBCL. Frail patients were those younger than 80 years with a frail profile at CGA or older than 80 years with an unfit profile. Treatment consisted of 4-6 courses of bendamustine $\left[90 \mathrm{mg} / \mathrm{m}^{2}\right.$ days (d)1-2] and rituximab $\left(375 \mathrm{mg} / \mathrm{m}^{2} \mathrm{~d} 1\right)$ administered every 28 days. Other main study end points were complete remission rate and the rate of extra-hematologic adverse events. Forty-nine patients were enrolled of whom 45 were confirmed eligible. Overall, 24 patients achieved a complete remission (53\%; 95\%CI: 38-68\%) and the overall response rate was $62 \%$ (95\%CI: $47-76 \%)$. The most frequent grade 3-4 adverse event was neutropenia (37.8\%). Grade 3-4 extra-hematologic adverse events were observed in 7 patients (15.6\%; 95\% CI: 6.5$29.5 \%$ ); the most frequent was grade 3 infection in 2 patients. With a median follow up of 33 months (range 1-52), the median progression-free survival was ten months (95\%CI: 7-25). The study shows promising activity and manageable toxicity profile of $\mathrm{BR}$ combination as first-line therapy for patients with DLBCL who are prospectively defined as frail according to a simplified CGA, as adopted in this trial (clinicaltrials.gov identifier: 01990144).

\section{Correspondence:}

stefano.luminari@ausl.re.it

Received: December 20, 2017.

Accepted: May 3, 2018.

Pre-published: May 10, 2018.

doi:10.3324/haematol.2017.186569

Check the online version for the most updated information on this article, online supplements, and information on authorship \& disclosures: www.haematologica.org/content/103/8/1345

\section{(C)2018 Ferrata Storti Foundation}

Material published in Haematologica is covered by copyright. All rights are reserved to the Ferrata Storti Foundation. Use of published material is allowed under the following terms and conditions:

https://creativecommons.org/licenses/by-nc/4.0/legalcode. Copies of published material are allowed for personal or internal use. Sharing published material for non-commercial purposes is subject to the following conditions:

https://creativecommons.org/licenses/by-nc/4.0/legalcode, sect. 3. Reproducing and sharing published material for commercial purposes is not allowed without permission in writing from the publisher. 


\section{Introduction}

Diffuse large B-cell lymphoma (DLBCL) is the most frequent non-Hodgkin lymphoma (NHL) and typically affects elderly patients; approximately $50 \%$ of the patients are older than 65 years and $15 \%$ are older than 80 years. ${ }^{1}$ More importantly, life expectancy has markedly improved over the past century and it is expected that the number of people older than 75 years will triple by the year 2030. ${ }^{2}$ As a consequence, the burden of age-related diseases, including DLBCL, is expected to increase in the near future.

$\mathrm{R}-\mathrm{CHOP}$ is the undisputed standard for the treatment of elderly patients up to 80 years of age. ${ }^{3}$ A remaining unmet need, however, is related to the treatment of elderly patients who, due to age or comorbidity, cannot be treated with full-dose standard treatment. In these subjects, the availability of less toxic regimens is strongly warranted but the decision-making process concerning therapeutic intervention should also include an accurate and objective patient selection. ${ }^{4}$ Bendamustine is an alkylating agent with properties of a purine analog and is approved for the treatment of chronic lymphocytic leukemia (CLL) and indolent NHL. Bendamustine was also studied in aggressive lymphomas; several phase II studies of BR (bendamustine in association with rituximab) in patients with relapsed refractory DLBCL showed promising efficacy with overall good tolerance of this regimen in the salvage setting..$^{5.7}$ The activity of BR was also tested with promising results in small phase II studies on untreated patients who were generally considered not eligible for standard $\mathrm{R}-\mathrm{CHOP} .8,9$

In 2014, the Fondazione Italiana Linfomi (FIL) started a phase II study to investigate the activity and the safety profile of a combination regimen of BR for the initial therapy of patients with DLBCL who were not eligible to receive standard anthracycline-based therapy. In contrast to other studies, we included patients who were prospectively classified as frail according to the Comprehensive Geriatric Assessment (CGA), ${ }_{10}^{10}$ and we evaluated a modified BR schedule. Based on available evidence, there is still no standard treatment for this subset of patients, complete response to therapy is approximately $15 \%,{ }^{11}$ and, even if rituximab is used, the median OS is approximately 20 months. ${ }^{12}$

\section{Methods}

\section{Study design and objectives}

This is a phase II open-label, non-randomized study to investigate activity and safety of BR combination therapy in elderly patients with DLBCL, prospectively defined as frail according to the CGA. The study was approved by the local ethics committees.

\section{Patient eligibility}

Eligible patients were elderly frail patients aged 70 years or over with a newly diagnosed, histologically proven DLBCL. All patients were prospectively evaluated by the CGA, as originally reported as part of pre-therapy assessment. Briefly, the CGA used was based on the assessment of Activities of Daily Living (ADL), Instrumental Activities of Daily Living (IADL), and Cumulative Illness Rating Scale-Geriatric (CIRS-G). Patients were classified as frail if the following criteria were met: in patients aged between 70 and 80 years, $\mathrm{ADL}<4$ or $\mathrm{IADL}<5$ or 1 grade 3 comorbidity or $>8$ grade 2 comorbidities were required; in patients older than 80 years, $\mathrm{ADL}>5$ or $\mathrm{IADL}>6$ or $5-8$ grade 2 comorbidities were required ${ }^{10}$ (Table 1). A full list of inclusion criteria and study procedures is available in the Online Supplementary Appendix.

\section{Treatment}

Patients received bendamustine $\left(90 \mathrm{mg} / \mathrm{m}^{2}\right.$, d1-2) combined with rituximab $\left(375 \mathrm{mg} / \mathrm{m}^{2}\right.$, d1) every 28 days. Patients with age-adjusted International Prognostic Index (aaIPI) equal to 0 and non-bulky disease received 4 cycles of BR followed by 2 cycles of rituximab. All other patients received 6 cycles of BR followed by 2 cycles of rituximab. Bendamustine was supplied for free by Mundipharma. The use of consolidation radiotherapy was allowed. Prophylaxis with valacyclovir and cotrimoxazole was mandatory. The use of granulocyte-colony stimulating factor (G-CSF) and erythropoietin was recommended.

\section{Statistical analysis}

The main study end point was the complete remission rate (CRR) that was calculated on the efficacy population (EP; i.e. patients receiving at least 2 courses of BR) using Cheson 1999 criteria. ${ }^{13}$ A further end point was the rate of grade 3-4 extrahematologic adverse events (eeAEs) calculated on the safety population (SP; i.e. all patients who have received at least one dose of study medication) using CTCAE v.4.0 (Common Terminology Criteria for Adverse Events). Secondary end points were progression-free survival (PFS) and overall survival (OS). ${ }^{14}$

The study was conducted according to a Simon 2-stage design. The null hypothesis ( $\mathrm{p} 0$ ) for CRR was set to $0.15 .{ }^{11}$ With a type I error of 0.05 and a type II error of 0.10 , and considering the alternative hypothesis ( $\mathrm{p} 1$ ) at 0.35 , at least 4 CRs were required after the enrollment of the first 19 cases. With the full enrollment of the 44 cases, at least 11 patients in CR were required to confirm activity of the combination to be promising. Considering a $10 \%$ drop-out rate, the final study enrollment was set at 49 patients.

Maximum tolerated toxicity rate for eeAEs was set at $30 \%$. Considering an alpha error of 0.05 , and according to the 2 -stage Simon design, the study had to be stopped if grade 3-4 events were observed in 12 or more patients out of the first 19 enrolled. With the enrollment of the planned 44 patients, a maximum number of 20 patients with grade 3-4 eeAEs were allowed.

\section{Results}

\section{Patients}

From February 2012 to February 2014, 49 patients were enrolled into the study by 24 Italian centers. Three patients

Table 1. Comprehensive Geriatric Assessment criteria for definition of fit, unfit and frail patients.

\begin{tabular}{lccc} 
& FII & UNFIT & FRAIL \\
ADL & 6 & $5^{*}$ & $\leq 4$ \\
IADL & 8 & $7-6^{*}$ & $\leq 5$ \\
\hline CIRS** & 0 score $=3-4$ & 0 score $=3-4$ & 1 score $=3-4$ \\
& $<5$ score $=2$ & $5-8$ score $=2$ & $>8$ score $=2$ \\
Age & & $\geq 80$ fit & $\geq 80$ unfit
\end{tabular}

ADL: Activity of Daily Living; IADL: Instrumental Activities of Daily Living; CIRS: Cumulative Illness Rating Scale. *Residual function. ${ }^{*}$ Scores are referred to severity of assessed comorbidities (as reported by Tucci et al..$^{10}$ ) 
were considered ineligible due to violation of inclusion criteria (one patient was not confirmed as frail, one did not undergo CGA evaluation, one case was HCV positive). One additional patient was excluded from the study analysis due to death before treatment start. The clinical characteristics of the remaining 45 patients are shown in Table 2. A full list of comorbidities with observed rates is provided in Online Supplementary Table S1.

\section{Feasibility and Efficacy}

Treatment was started in 45 eligible patients. Nine patients were at low risk (aaIPI 0 and non-bulky disease) and had to receive $4 \mathrm{BR}$ cycles followed by two doses of rituximab. All the other patients $(n=36)$ were at high risk and had to receive all 6 BR cycles followed by two doses of rituximab. Overall, 25 patients received all planned bendamustine doses (55\%); 7 of 9 at low risk, and 18 of 36 at high risk. Treatment was discontinued due to progressive disease (12 patients), eeAEs (8 patients) and physician's decision (7 patients). Four non-bulky patients ( 3 with stage I-II, 1 with stage IV) received consolidation radiotherapy. In 4 high-risk cases, treatment was interrupted before administration of the 2 consolidation doses of rituximab. Three additional patients interrupted treatment after cycle $4(\mathrm{n}=1)$ and $5(\mathrm{n}=2)$ due to physician's decision. The calculated administered dose intensity for rituximab and bendamustine was 0.990 (25-75 percentiles: 0.901-1.016), and 0.996 (25-75 percentiles: 0.877-1.017).

The efficacy analysis was based on all 45 cases. After the enrollment of the first 19 patients, 5 CRs were observed and the accrual to study stage 2 opened. Overall, 24 patients achieved a CR at the end of treatment $(53 \%$; 95\%CI: $38-68 \%$ ), 4 patients achieved a partial remission (PR), one patient had a stable disease (SD), 13 patients had progressive disease (PD), and in 3 patients response was not evaluable (due to AEs: pelvic fracture, pneumonia, heart attack). The overall response rate (ORR) was $62 \%$ (95\% CI: $47-76 \%)$. The observed CRR was higher than that initially required for efficacy assessment by the study design (Table 3).

\section{Safety}

The safety analysis was available for all 45 eligible patients and for 244 cycles. Thirty-five grade 3-4 AEs were reported in 23 patients (51.1\%; 95CI: 35.8-66.3\%); the most frequent grade 3-4 AE was neutropenia with 17 events $(37.8 \%)$ (Table 4). Though not mandatory, G-CSF was used in 26 patients (58\%; 95CI: $42-72 \%$ ).

The rate of grade 3-4 eeAE was monitored during stage 1 and stage 2 of the study. Overall, grade 3-4 eeAEs were reported in 7 patients (15.6\%; 95CI: $6.5-29.5 \%$ ) and grade 3-4 hematologic AEs were reported in 21 cases (46.7\%; 95CI: 31.7-62.1\%). The most frequent grade 3-4 eeAE was infection ( $\mathrm{n}=2$ patients, $4.4 \%$ ); no grade 4 infections were reported. A detail of all reported AEs is provided in Table 4. Both for study stage I and stage II, the rate of grade 3-4 eeAEs never fell beyond the maximum rate allowed.

\section{Survival analysis}

The median follow up was 33 months (range 1-52). Thirty-two patients experienced PD, including $13 \mathrm{PD}$ at the end of induction therapy, 12 relapses, and 7 deaths for causes unrelated to lymphoma (pneumonia, heart failure, hepatocarcinoma, neurological disorder, respiratory disorder, cachexia, unknown). The 2-year PFS was $38 \%$
(95\% CI: $24-51 \%$ ) and the median PFS was 10 months (95\%CI: $7-25 \%$ ) (Figure 1).

Overall, 24 patients died: 8 during treatment and 16 during follow up. Cause of death was lymphoma progression in 14 patients $(58 \%)$, and unrelated causes in 8 patients (the 7 deaths reported above, plus one patient who died due to secondary acute myeloyd leukemia after lymphoma progression). Two-year OS was $51 \%$ (95\% CI: 35 $65 \%)$ and median OS was 30 months (95\% CI: 10-NA). The results of the CGA scales used during patients' baseline assessment were correlated with OS and PFS in univariate analysis, and no association was found (Table 5). In addition, no association was found between results of CGA scales and safety results (data not shown).

\section{Discussion}

We report the results of a phase II study to investigate the activity and toxicity of a combination regimen of ritu$x i m a b$ and bendamustine for the initial treatment of patients with DLBCL who were classified as frail based on

Table 2. Baseline characteristics of patients eligible for the study $(n=45)$.

\begin{tabular}{lccc} 
& Status & Missing & N (\%) \\
Sex & Male & - & $26(58)$ \\
Age (years) & Median (range) & - & $81(71-89)$ \\
\hline $\mathrm{Hb}(\mathrm{g} / \mathrm{dL})$ & Median (range) & - & $12.9(7.8-16.1)$ \\
Stage & I & - & $7(16)$ \\
& II & & $10(23)$ \\
& III & & $6(14)$ \\
ECOG PS & IV & 1 & $22(48)$ \\
ENS & $>1$ & - & $16(36)$ \\
\hline LDH & $>1$ & - & $11(24)$ \\
IPI & $>$ ULN & 1 & $16(36)$ \\
CGA & $3-5$ & - & $25(57)$ \\
& Unfit with age $\geq 80$ years & & $35(78)$ \\
frail & & 4 & $10(22)$
\end{tabular}

\section{LVEF (\%) $\quad$ Median (range) $\quad 4 \quad 60$ (43-70)}

Hb: hemoglobin: ECOG PS: Eastern Cooperative Oncology Group Performance Status; ENS: extra nodal site; LDH: lactate dehydrogenase; ULN:upper limit of normal; IPI: International Prognostic Index; CGA: comprehensive geriatric assessment; LVEF: left ventricular ejection fraction.

Table 3. Response after planned rituximab plus bendamustine treatment.

\begin{tabular}{lccc} 
Response & $\begin{array}{c}\mathrm{R} 8+\mathrm{B} 6(\mathrm{n}=36) \\
\mathbf{N}(\%)\end{array}$ & $\begin{array}{c}\mathrm{R} 6+\mathrm{B} 4(\mathrm{n}=9) \\
\mathbf{N}(\%)\end{array}$ & $\begin{array}{c}\text { Total }(\mathrm{n}=45) \\
\mathbf{N}(\%, \% 95 \mathrm{C})\end{array}$ \\
$\mathrm{CR}$ & $19(53)$ & $5(56)$ & $24(53 ; 38-68)$ \\
$\mathrm{PR}$ & $4(11)$ & - & $4(9 ; 2-21)$ \\
\hline $\mathrm{ORR}$ & & & $28(62 ; 47-76)$ \\
$\mathrm{SD}$ & $1(3)$ & - & $1(2 ; 0-12)$ \\
\hline $\mathrm{PD}$ & $9(25)$ & $4(44)$ & $13(29 ; 16-44)$ \\
Not assessed & $1(3)$ & - & $1(2 ; 0-12)$ \\
\hline Death in treatment * & $2(6)$ & - & $2(4 ; 1-15)$ \\
\hline
\end{tabular}

*Dead for heart failure and pneumonia. R8+B6: rituximab for 8 cycles and bendamustine for 6 cycles; R6+B4: Rituximab for 6 cycles and bendamustine for 4 cycles; $n / \mathrm{N}$ : number; CI: Confidence Interval; CR: complete remission; PR: partial remission; ORR: overall response rate; SD: stable disease; PD: progression disease. 
a CGA. With the observed $53 \%$ CR rate, and with the $15.6 \%$ rate of grade $3-4$ eeAEs, the study met its primary objectives and shows the promising activity of the combination in a difficult-to-treat patient population

Besides the analysis of the main study end points (response rate and safety), we acknowledge that the small sample size of this study does not allow any firm conclusions to be drawn on the interpretation of secondary end points, and in particular on PFS and OS data. Moreover, the lack of a centralized histology review and cell of origin analysis does not allow us to present any hypothesis on the differential activity of bendamustine and rituximab combination among DLBCL subtypes.

Since the activity of bendamustine in DLBCL was first documented by Weidmann et al. ${ }^{15}$ several phase II and retrospective studies have been published to assess the activity and the safety profile of this combination. In the first prospective studies on relapsed refractory patients, bendamustine was used at higher doses $\left(120 \mathrm{mg} / \mathrm{m}^{2} / \mathrm{d}\right)$ and with a shorter interval between cycles. ${ }^{5.7}$ In these trials, the ORR ranged between $46 \%$ and $63 \%$ (CR 15-37\%) and the median PFS between 3.6 and 6.7 months (Table 6). Park et al. ${ }^{8}$ recently published the results of a small phase II trial of bendamustine at $120 \mathrm{mg} / \mathrm{m}^{2}$ in combination with rituximab in untreated older patients ( $>65$ years) who were poor candidates for R-CHOP. Among the 23 enrolled patients, the median age was 80 years, the ORR and the CRR were $78 \%$ and $52 \%$, respectively, but the median PFS and OS were only 5.4 and 10 months, respectively. In our study, in consideration of the patients' age and frail status, we opted for a regimen with bendamustine at a lower dose of $90 \mathrm{mg} / \mathrm{m}^{2}$ combined with standard rituximab doses, and administered every four weeks. Looking at our results, this choice did not seem to significantly reduce treatment activity compared to more intense published BR regimens. Conversely, the adoption of a less intense BR combination was well tolerated and was a good choice for patients who were prospectively identi- fied as frail. Comparing our data with those from prior phase II reports, it should also be acknowledged that Park et al. did not include stage I disease and had more poor performance status patients, while Weidmann et al. had more early stage patients, and a higher median age (85 years). Our data should also be compared with a previous analysis of 99 elderly frail patients with DLBCL who were analyzed in a prospective observational study.12 The

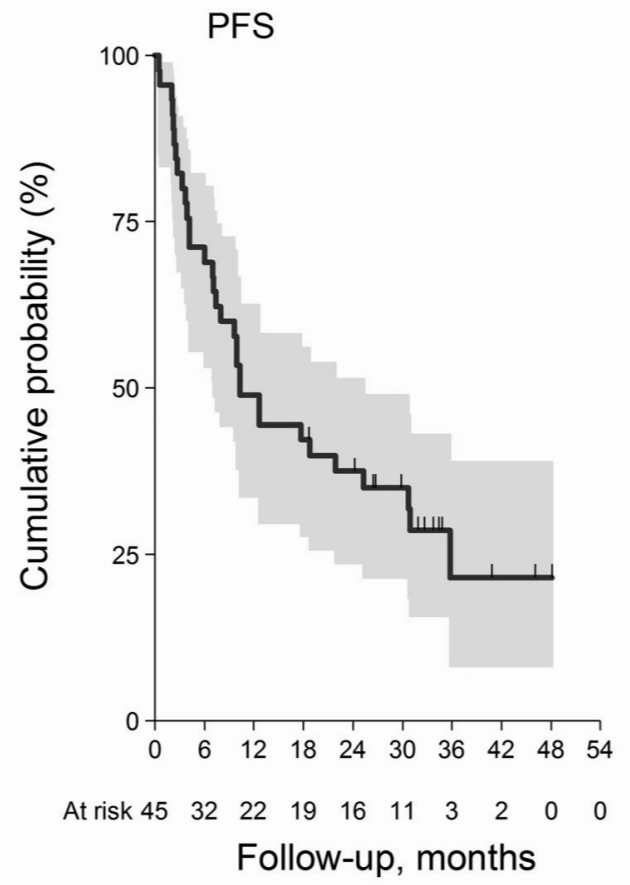

Figure 1. Estimated progression-free survival (PFS) with $95 \%$ confidence interval (gray area).

Table 4. Overall toxicities according to CTCAE v.4.0 categories with grade.

\begin{tabular}{|c|c|c|c|c|c|c|}
\hline & \multicolumn{2}{|c|}{ All grades } & \multicolumn{2}{|c|}{ Grade 3} & \multicolumn{2}{|c|}{ Grade 4} \\
\hline & $\mathbf{n}$ & $\%$ & n & $\%$ & n & $\%$ \\
\hline Anemia & 20 & 44.4 & 1 & 2.2 & 0 & 0.0 \\
\hline Leucopenia & 19 & 42.2 & 3 & 6.7 & 2 & 4.4 \\
\hline Neutropenia & 29 & 64.4 & 8 & 17.8 & 9 & 20.0 \\
\hline Thrombocytopenia & 20 & 44.4 & 4 & 8.9 & 0 & 0.0 \\
\hline Febrile neutropenia & 3 & 6.7 & 0 & 0.0 & 1 & 2.2 \\
\hline Infections & 9 & 20.0 & 2 & 4.4 & 0 & 0 \\
\hline Fever & 2 & 4.4 & 0 & 0.0 & 0 & 0.0 \\
\hline Cardiac disorders & 4 & 8.9 & 1 & 2.2 & 1 & 2.2 \\
\hline Gastrointestinal disorders & 14 & 31.1 & 0 & 0.0 & 0 & 0.0 \\
\hline General disorders and & & & & & & \\
\hline administration site conditions* & 5 & 11.1 & 1 & 2.2 & 0 & 0.0 \\
\hline Hepatobiliary disorders & 2 & 4.4 & 0 & 0.0 & 0 & 0.0 \\
\hline Metabolism and nutrition disorders & 3 & 6.7 & 1 & 2.2 & 0 & 0.0 \\
\hline Nervous system disorders & 4 & 8.9 & 0 & 0.0 & 0 & 0.0 \\
\hline Renal and urinary disorders & 4 & 8.9 & 1 & 2.2 & 0 & 0.0 \\
\hline Skin and subcutaneous tissue disorders & 9 & 20.0 & 0 & 0.0 & 0 & 0.0 \\
\hline Vascular disorders & 2 & 4.4 & 0 & 0.0 & 0 & 0.0 \\
\hline Other (specify) $* *$ & 4 & 8.9 & 0 & 0.0 & 0 & 0.0 \\
\hline
\end{tabular}

CTCAE: Common Terminology Criteria for Adverse Events; $n$ : number. *Asthenia; laboratory abnormalities; fever. ** Other - Grade 1: epistaxis and flu; Grade 2: accidental fall and cough. 
majority of frail subjects of this study were treated with rituximab-containing therapy $(39 \%)$ and 31 of them also received an anthracycline-containing regimen. Considering only the patients who were treated with chemo-immunotherapy, the 2-year and median OS were $48 \%$ and 20 months, respectively. These data compare favorably with those observed in the current study that used a more strict definition of frailty (i.e. unfit patients with intermediate profile at CGA were not included) and were obtained without the use of doxorubicin.

When this trial was started, data from a large observational study on 173 consecutive elderly patients with DLBCL were already available. These suggested that the use of chemotherapy regimens with curative intent were not able to improve patients' survival compared to palliative therapies for the subgroup of patients who were classified as frail according to the same CGA that we adopted in this trial..$^{10}$ Based on this observation, we considered frail those patients ineligible to receive anthracycline-containing regimens, also if administered at lower doses, and identified an unmet need in the search of active therapies in this subset of patients. Unfortunately, lacking a randomized comparison, we cannot draw any conclusion on the relative efficacy of the $\mathrm{BR}$ regimen in comparison with other immunochemotherapy options. Due to its favorable safety profile, however, the use of a BR combination seems an excellent option and should be set as a reference to identify more effective treatment strategies in future trials. Regarding safety, the recent experience with BR in follicular lymphoma reported an increase in toxicities; with the limitations of a small number of patients, in our population, BR was manageable and safe; the two deaths due to second malignancies is not an unexpected finding in a very elderly population.

In order to further improve these results, additional efforts should be made to attempt to increase the response rate and to prolong the short duration of response. New drugs such as lenalidomide and ibrutinib have shown activity against DLBCL, are both associated with an excellent safety profile,,$^{16,17}$ and could be used to improve the results with an acceptable toxicity. A phase II trial of rituximab in association with a lenalidomide combination in elderly frail patients is currently being conducted by our group and actively recruiting patients (clinicaltrials.gov identifier: 02955823). In addition, the published results of the REMARC trial has demonstrated, for the first time after several unsuccessful attempts with other drugs, ${ }^{18}$ that a maintenance therapy with lenalidomide in elderly patients with DLBCL who responded to initial immunochemother- apy is associated with a reduced risk of disease progression compared to observation. ${ }^{19}$ Finally, our study is part of a larger project for elderly patients with DLBCL for whom a preliminary CGA is required to define patient fitness status and to adapt treatment goals accordingly. Patients prospectively enrolled in this so called "elderly project" are evaluated by a simplified version of the CGA ${ }^{20}$ and are categorized into one of three groups: fit, unfit and frail (clinicaltrials.gov identifier: 02364050). Fit patients are then offered a standard R-CHOP treatment with curative intent, and unfit patients are considered better candidates for adapted R$\mathrm{CHOP}$ regimens with reduced drug doses to achieve a cure with reduced toxicity. Finally, no clear benefit was observed for frail patients treated with curative intent compared with those treated with palliative intent, and there was no standard or reference regimen. ${ }^{21}$ To the best of our knowledge, the elderly project is the first attempt to try to objectify the treatment approach to elderly patients with lymphoma and to promote clinical research in this population. With our study, and with the adoption of a prospective definition of patient fitness, we have been able to show that, also in the population of frail patients, the use of a low toxicity regimen allows a cure of the lymphoma to be achieved in a good proportion of patients. These data, along with the adopted CGA evaluation, help

Table 5. Association of age, geriatric scales and IPI with OS and PFS (univariate analysis).

\begin{tabular}{|c|c|c|c|c|}
\hline Variable & $\begin{array}{c}1 \mathrm{yr}-0 \mathrm{~S} \% \\
\text { (95C) }\end{array}$ & $\begin{array}{c}\text { Log-rank } \\
P\end{array}$ & $\begin{array}{c}1 y r \text { PFS \% } \\
\text { (95\%Cl) }\end{array}$ & $\begin{array}{c}\text { Log-rank } \\
P\end{array}$ \\
\hline $\begin{array}{l}\text { Age } \\
\leq 80 \\
>80\end{array}$ & $\begin{array}{l}68(29-88) \\
62(44-76)\end{array}$ & 0.694 & $\begin{array}{l}60(25-83) \\
46(29-61)\end{array}$ & 0.685 \\
\hline $\begin{array}{l}\mathrm{ADL} \\
6 \\
5 \\
0-4\end{array}$ & $\begin{array}{l}70(48-85) \\
50(21-74) \\
63(23-86)\end{array}$ & 0.335 & $\begin{array}{c}56(35-73) \\
25(6-50) \\
62(23-86)\end{array}$ & 0.536 \\
\hline $\begin{array}{l}\text { IADL } \\
8 \\
6-7 \\
0-5\end{array}$ & $\begin{array}{c}33(5-68) \\
71(49-85) \\
62(32-82)\end{array}$ & 0.350 & $\begin{array}{c}33(5-68) \\
56(35-73) \\
43(18-66)\end{array}$ & 0.020 \\
\hline $\begin{array}{c}\text { IPI } \\
1-2 \\
3-5\end{array}$ & $\begin{array}{l}77(50-91) \\
52(31-69)\end{array}$ & 0.531 & $\begin{array}{l}58(33-76) \\
40(21-58)\end{array}$ & 0.581 \\
\hline
\end{tabular}

yr: year; CI: Confidence Interval; ADL: Activities of Daily Living; IADL: Instrumental Activities of Daily Living; IPI: International Prognostic Index; OS: overall survival; PFS: progression-free survival.

Table 6. Summary of prospective studies of bendamustine in association with rituximab in diffuse large B-cell lymphoma.

\begin{tabular}{|c|c|c|c|c|c|c|c|c|}
\hline Author & $\begin{array}{c}\text { Year } \\
\text { (medAge) }\end{array}$ & Patients & Phase & $\begin{array}{c}\text { Benda dose } \\
\mathrm{mg} / \mathrm{m}^{2}\end{array}$ & Days & CRR\% & ORR\% & $\begin{array}{l}\text { mPFS } \\
\text { months }\end{array}$ \\
\hline Ohmachi et $a l^{6}$ & 2013 & $59(65)$ & $\mathrm{R} / \mathrm{R}$ & 120 & 21 & 37.7 & 62.7 & 6.7 \\
\hline Vacirca et al. ${ }^{7}$ & 2014 & $48(74)$ & $\mathrm{R} / \mathrm{R}$ & $90 / 120^{*}$ & 28 & 15.3 & 45.8 & 3.6 \\
\hline Weidmann et al. ${ }^{9}$ & 2011 & $13(85)$ & Untr. & 120 & 21 & 54 & 69 & 7.7 \\
\hline Park et al..$^{8}$ & 2016 & $23(80)$ & Untr. & 120 & 21 & 52 & 78 & 5.4 \\
\hline Current report & 2016 & $45(81)$ & Untr. & 90 & 28 & 53 & 62 & 10 \\
\hline
\end{tabular}

*Bendamustine (Benda) dosage $90 \mathrm{mg} / \mathrm{m}^{2}$ was administered for the first 2 patients on study. Following the US FDA approval, the dosage was amended to $120 \mathrm{mg} / \mathrm{m}^{2}$. R/R: relapsed/refractory; medAge: median age; Untr: untreated; CRR: complete remission rate; ORR: overall response rate; mPFS: median progression-free survival. 
to define new reference points for future therapeutic development in an otherwise highly heterogeneous and difficult-to-treat population.

In conclusion, considering our results, and the other available data discussed above, we believe that the combination of bendamustine and rituximab, even if not curative, is a good option for the treatment of elderly frail patients with DLBCL, also when used at reduced doses and administered at 4-week intervals as in our study. Treatment of elderly frail patients remains a challenge for the clinician, and the choice of treatment should be indi- vidualized considering all available options and through an accurate assessment of the risk-benefit ratio for each single patient. At least as important as the choice of treatment, a concerted effort should be made to adopt validated tools to assess patient fitness status before treatment start and to adapt treatment goals accordingly. In this context, we recommend the use of the simplified version of the CGA as used in this study.

\section{Funding}

This work was supported by Mundipharma Pharmaceuticals.

\section{References}

1. Bellera C, Praud D, Petit-Moneger A, McKelvie-Sebileau P, Soubeyran P, Mathoulin-Pelissier S. Barriers to inclusion of older adults in randomised controlled clinical trials on Non-Hodgkin's lymphoma: a systematic review. Cancer Treat Rev. 2013;39(7):812-817.

2. Jemal A, Siegel R, Ward E, Murray T, Xu J, Thun MJ. Cancer statistics, 2007. CA Cancer J Clin. 2007:57(1):43-66.

3. Coiffier B, Lepage E, Briere J, et al. CHOP chemotherapy plus rituximab compared with $\mathrm{CHOP}$ alone in elderly patients with diffuse large-B-cell lymphoma. N Engl J Med. 2002;346(4):235-242.

4. Peyrade F, Jardin F, Thieblemont C, et al. Attenuated immunochemotherapy regimen (R-miniCHOP) in elderly patients older than 80 years with diffuse large B-cell lymphoma: a multicentre, single-arm, phase 2 trial. Lancet Oncol. 2011;12(5):460468.

5. Ogura M, Ando K, Taniwaki M, et al. Feasibility and pharmacokinetic study of bendamustine hydrochloride in combination with rituximab in relapsed or refractory aggressive B cell non-Hodgkin's lymphoma. Cancer Sci. 2011;102(9):1687-1692.

6. Ohmachi $K$, Niitsu $N$, Uchida $T$, et al. Multicenter phase II study of bendamustine plus rituximab in patients with relapsed or refractory diffuse large B-cell lymphoma. J Clin Oncol. 2013;31(17):21032109.

7. Vacirca IL, Acs PI, Tabbara IA, Rosen PI, Lee P, Lynam E. Bendamustine combined with rituximab for patients with relapsed or refractory diffuse large B cell lymphoma. Ann Hematol. 2014;93(3):403-409.
8. Park SI, Grover NS, Olajide O, et al. A phase II trial of bendamustine in combination with rituximab in older patients with previously untreated diffuse large B-cell lymphoma. Br J Haematol. 2016;175(2): 281-289.

9. Weidmann E, Neumann A, Fauth F, et al. Phase II study of bendamustine in combination with rituximab as first-line treatment in patients 80 years or older with aggressive B-cell lymphomas. Ann Oncol. 2011:22(8):1839-1844

10. Tucci A, Martelli M, Rigacci L, et al. Comprehensive geriatric assessment is an essential tool to support treatment decisions in elderly patients with diffuse large B-cell lymphoma: a prospective multicenter evaluation in 173 patients by the Lymphoma Italian Foundation (FIL). Leuk Lymphoma. 2015;56(4):921-926.

11. Monfardini S, Aversa SM, Zoli V, et al. Vinorelbine and prednisone in frail elderly patients with intermediate-high grade nonHodgkin's lymphomas. Ann Oncol. 2005:16(8):1352-1358

12. Merli F, Luminari S, Rossi G, et al. Outcome of frail elderly patients with diffuse large Bcell lymphoma prospectively identified by Comprehensive Geriatric Assessment: results from a study of the Fondazione Italiana Linfomi. Leuk Lymphoma. 2014;55(1):38-43.

13. Cheson BD, Horning SJ, Coiffier B, et al. Report of an international workshop to standardize response criteria for nonHodgkin's lymphomas. NCI Sponsored International Working Group. J Clin Oncol. 1999;17(4):1244

14. Cheson BD, Pfistner B, Juweid ME, et al. Revised response criteria for malignant lymphoma. J Clin Oncol. 2007;25(5):579586.
15. Weidmann E, Kim SZ, Rost A, et al. Bendamustine is effective in relapsed or refractory aggressive non-Hodgkin's lymphoma. Ann Oncol. 2002;13(8):1285-1289.

16. Hitz F, Zucca E, Pabst T, et al. Rituximab, bendamustine and lenalidomide in patients with aggressive B-cell lymphoma not eligible for anthracycline-based therapy or intensive salvage chemotherapy - SAKK 38/08. Br J Haematol. 2016;174(2):255-263.

17. Maddocks K, Christian B, et al. A phase $1 / 1 b$ study of rituximab, bendamustine, and ibrutinib in patients with untreated and relapsed/refractory non-Hodgkin lymphoma. Blood. 2015;125(2):242-248.

18. Habermann TM, Weller EA, Morrison VA, et al. Rituximab-CHOP versus $\mathrm{CHOP}$ alone or with maintenance rituximab in older patients with diffuse large B-cell lymphoma. J Clin Oncol. 2006;24(19):31213127.

19. Thieblemont C, Tilly H, Gomes da Silva M, et al. Lenalidomide Maintenance Compared With Placebo in Responding Elderly Patients With Diffuse Large B-Cell Lymphoma Treated With First-Line Rituximab Plus Cyclophosphamide, Doxorubicin, Vincristine, and Prednisone. J Clin Oncol. 2017;35(22):2473-2481.

20. Spina M, Balzarotti M, Uziel L, et al Modulated chemotherapy according to modified comprehensive geriatric assessment in 100 consecutive elderly patients with diffuse large B-cell lymphoma. Oncologist. 2012;17(6):838-846.

21. Tucci A, Ferrari S, Bottelli C, Borlenghi E, Drera M, Rossi G. A comprehensive geriatric assessment is more effective than clinical judgment to identify elderly diffuse large cell lymphoma patients who benefit from aggressive therapy. Cancer. 2009;115(19):4547-4553. 